\title{
L'enseignement de la distinction passé composé/imparfait aux apprenants anglophones de FLE : un test de deux explications dans la salle de classe
}

\author{
Melissa Whatley \\ The University of Georgia \\ melwhat7@gmail.com
}

\section{Introduction : L'aspect et les explications des manuels de FLE}

Le système aspectuel du français, plus particulièrement la distinction entre le passé composé (PC) et l'imparfait (IMP), est un des adversaires les plus redoutables pour les apprenants anglophones puisque leur langue native ne connaît pas de telle distinction. Les explications de cette distinction aspectuelle ne manquent pas dans les manuels de FLE ni dans les articles de linguistique mais il manque des expériences pour tester l'effet de ces explications sur l'apprentissage par des apprenants. L'objectif de la présente étude est donc de mettre à l'épreuve dans la salle de classe de FLE aux États-Unis deux explications de la distinction entre le PC et l'IMP, une proposée par le manuel Chez nous (Pons et alii, 2006) et l'autre par des linguistes (Dansereau, 1987 ; Frantzen, 1995).

Vendler (1967) offre une explication de l'aspect qui partage les verbes en quatre catégories : les activités, les achèvements, les accomplissements et les états (Vendler 1967: 102- 103) pendant que Weinrich (1964) explique ces deux alternatives dans les langues romanes en référence au premier plan (PC) et l'arrière-plan (IMP) (Weinrich 1964: 73). Comrie (1976:12) décrit une situation perfective, à laquelle on réfère en français par le PC, comme «a situation viewed in its entirety, without regard to internal temporal constituency ». Une action imparfaite, par contre, représentée par l'IMP en français, est une action progressive ou continue (Comrie 1976: 12). Les locuteurs peuvent aussi manipuler le contraste aspectuel pragmatiquement. Lunn (1985) signale que le point de vue du locuteur est très pertinent pour le choix d'aspect. Si le locuteur voit une action comme une action perfective, le locuteur marque le verbe comme perfectif tandis que si le locuteur la voit comme imperfective, il marque le verbe en conséquence. De cette perspective on apprend que ce n'est pas l'action elle-même qui est perfective ou imperfective plutôt cette distinction est déterminée par la perspective du locuteur. Bien sûr, il y a quelques verbes qui confèrent l'aspect perfectif plus souvent que des autres, mais le point de vue du locuteur joue aussi un rôle dans le choix de l'aspect. Comme le choix entre le PC et l'IMP dépend au moins partiellement du point de vue du locuteur et non pas seulement de l'action elle-même, les apprenants ont de la difficulté à déterminer si un certain événement s'exprimerait au PC ou à l'IMP.

Comme le système aspectuel du français avec le PC et l'IMP ne correspond pas très bien à celui de l'anglais, qui a un passé simple et un passé progressif (Smith, 1983), les apprenants ont besoin d'aide pour comprendre l'emploi du PC et de l'IMP. Les manuels de FLE essaient donc de leur donner des règles pour faciliter le choix entre le PC et l'IMP. Selon Blyth (1997 : 53-55) deux raisons expliquent la grande influence de ces explications, surtout aux premiers niveaux universitaires. La première est l'inexpérience des professeurs de ces cours, qui sont souvent des étudiants gradués qui enseignent pour la première fois. "Textbooks have a particularly strong influence on inexperienced teachers, shaping their teaching practices and even their beliefs about language learning. Often an inexperienced teacher's metalinguistic knowledge is simply a reflection of the textbook's grammatical explanations. (Blyth, 1997 : 53-54) » La deuxième est la dépendance des étudiants aux manuels qu'ils utilisent pour faire les devoirs et les activités en classe et pour se préparer aux examens. Il n'est pas surprenant que les manuels aient beaucoup d'influence sur les apprenants de FLE. 
Dans sa critique des manuels des niveaux élémentaire, intermédiaire, et avancé pour enseigner l'espagnol, dont le prétérit correspond assez bien au PC du français et l'imparfait à l'IMP, Frantzen (1995: 145) maintient que « Textbooks are partially to blame for the difficulty students have with this concept because in their attempts at capturing the preterit/imperfect distinction, they often provide confusing, unreliable, and even inaccurate explanations. » Elle montre des exemples des règles des manuels qui sont valables dans quelques situations seulement ou qui sont totalement invalidées par les emplois des locuteurs natifs.

Dansereau (1987 : 33) conclut que la confusion des étudiants débutants du français provoqué par le système aspectuel de cette langue est due aux «vague, incomplete, contradictory, and generally poor explanations found in most beginning textbooks. » Elle signale deux problèmes avec les explications traditionnelles de l'aspect: "First, they are ambiguous and confusing : in every single case, exceptions to the definition are easily pointed out. Second, some of the statements lead the student to believe wrongly that the difference between the two tenses is semantic, when in fact it is aspectual (34). » Le manuel Chez nous (Pons et alii 2006 : 251) offre les deux règles suivantes pour l'IMP qui prêtent flanc des critiques de Dansereau :

1. To describe the weather: Il pleuvait et il faisait froid.

\section{To express feelings or describe emotions: Nous avions froid.}

Ces phrases sont certes possibles puisque le temps et les émotions peuvent être représentés comme des états imperfectifs, mais il est tout aussi possible de dire il a plu et nous avons eu froid. Quelques pages avant la description de l'IMP dans le même manuel se trouve la phrase (3) où le temps est exprimé par le PC :

3. Comme il a fait très beau, on a même fait un pique-nique au bord du lac. (247)

Quand les exemples du manuel contredisent ses propres règles, il n'est pas surprenant que les étudiants soient confus.

Dansereau (1987 : 37) offre une explication qu'elle appelle aspectuelle comme alternative à l'explication du manuel qui brille par sa simplicité : « The imparfait is used to relate what the conditions were/what was going on ("Quelles étaient les conditions? 》) and the passé composé to relate what happened/what happened next ("Qu'est-ce qui s'est passé ? »)». Cette explication rappelle l'explication de Weinrich (1964) qui associe le premier plan à $\mathrm{PC}$ et l'arrière plan à IMP. Dansereau prétend que cette explication atteint de bons résultats dans sa propre salle de classe, mais elle ne fournit pas de preuve quantitative qui puisse nous convaincre définitivement que cette explication soit préférable aux autres explications des manuels. L'objectif de la présente étude est donc de mettre à l'épreuve dans la salle de classe de FLE aux États-Unis deux explications de la distinction entre le PC et l'IMP. Une est une explication aspectuelle tandis que l'autre est une explication typique du manuel.

\section{Méthodologie}

Les apprenants qui ont participé à cette étude sont des apprenants anglophones de niveau débutant (deuxième semestre d'étude) dans une université dans le Sud-est des États-Unis. Suivant l'objectif de cette étude les apprenants ont été divisés en deux groupes dont un groupe expérimental qui a reçu comme enseignement une explication aspectuelle $(\mathrm{N}=9)$ en plus de l'explication du manuel du cours Chez nous et un autre groupe témoin qui n'a reçu que l'explication du manuel $(\mathrm{N}=18)$. L'explication aspectuelle proposée au groupe expérimental, adaptée de Dansereau (1987 : 37) et Frantzen (1995 : 147), est la suivante :

Both the passé composé and the imparfait express past actions and states, and all verbs can be used in either form. They serve different functions in a narrative, however. The passé composé is used to relate what happened/what happened next (Qu'est-ce qui s'est passé?). The imparfait is used to relate what the conditions were/what was going on (Quelles étaient les conditions?) 
Imparfait:

The imparfait is used to describe habitual actions or states.

Quand j'étais petite, j'allais à l'école chaque jour.

D'habitude la famille allait au parc le dimanche.

The imparfait is also used to set the scene, create an atmosphere, or relate what the conditions were when something else happened (the something else that happened is told using the passé composé).

Hier, j'étais très fatiguée - il pleuvait et je voulais dormir.

Ils quittaient l'église quand il a commencé à pleuvoir.

Passé composé

The passé composé can be used to focus on either the beginning or the end of an action or state.

Bruno a terminé ses études en juin.

Jean a habité à New York pour 4 ans.

Nous avons mangé à $20 \mathrm{~h}$ hier soir.

The passé composé can also be used to focus on an action or state as something that simply happened.

Elle est née le jeudi 9 mai 1991.

Elle a visité le Canada deux fois.

Plusieurs chercheurs soutiennent cette explication de l'aspect. On a déjà mentionné l'étude de Lunn (1985) qui se concentre sur le point de vue du locuteur qui influence le choix de l'aspect en espagnol. Labelle (1987) discute les rôles différents du PC et de l'IMP dans le discours tandis que Guitart (1978) discute quelques uns des mêmes principes que ceux cités ci-dessous chez les locuteurs natifs.

L'explication que l'autre groupe a reçue, et qui est dans le manuel du cours des deux groupes, est l'explication suivante du manuel Chez nous (2006) :

Tableau 1 : Explication de l'aspect dans le manuel Chez nous (Pons et alii 2006 : 192-93, 251, 270, 280)

\begin{tabular}{|l|l|}
\hline Explication du PC & Explication de l'IMP \\
\hline - actions completed in the past & - to make suggestions \\
- an event in the past has been completed & - to indicate time \\
- recount actions or events that move the story & - to indicate weather \\
forward & - to describe people and places \\
- What happened? & - to express feelings or describe emotions \\
& - habitual actions in the past \\
& - background information \\
& - What were the circumstances? What was \\
& going on? \\
& - time, weather, ongoing actions, physical \\
& characteristics, psychological states, intentions, \\
& feelings, thoughts \\
\hline
\end{tabular}

Ici on voit deux explications assez différentes. La première, qui est aspectuelle, donne plus de liberté à l'apprenant parce que cette distinction est basée sur son propre point de vue. Avec cette explication, on s'attend à ce que l'apprenant réfléchisse plus sur comment il voit les situations dans le passé. Si l'étudiant suit l'explication aspectuelle, notre hypothèse est qu'il emploiera l'IMP pour parler des actions à l'arrière-plan, des conditions, et des actions habituelles. De l'autre côte, il emploiera le PC pour les actions d'une narration, les choses qui se sont passées, et pour le début et la fin des actions ou des états. Le danger de cette explication, comme Westfall et Foerster (1996 : 550) signalent, est que les apprenants pensent que les deux formes verbales sont complètement interchangeables, et que tous les deux sont possibles dans toutes les situations. 
Par contre, les étudiants qui ne reçoivent que l'explication de Chez nous ont beaucoup plus de choix en ce qui concerne l'aspect. En ce qui concerne l'IMP, on s'attend à ce qu'ils emploient cette forme verbale pour décrire les gens, les endroits, et la scène en général, pour donner l'information de l'arrière plan, et pour parler des émotions. Pour le PC, on s'attend à ce qu'ils l'utilisent pour raconter les événements d'une narration et pour parler des actions complétées dans le passé. Le défaut le plus grave de cette explication est que ces règles font croire que la distinction aspectuelle dépend du sens du verbe. Par exemple, cette explication pourrait donner l'impression que si un verbe décrit une action qui est le plus souvent habituelle, le verbe devrait donc toujours être à l'imparfait. Comme Dansereau (1987: 34) dit, «some of the statements lead students to believe wrongly that the difference between the two tenses is semantic, when in fact it is aspectual. » L'erreur des règles de Chez nous est l'opposée de celle de l'explication aspectuelle. Tandis que l'explication aspectuelle donne l'impression que les deux aspects sont interchangeables et se déterminent selon le point de vue du locuteur, l'explication du manuel donne l'impression que, pour chaque situation, on peut employer soit le PC soit l'IMP, et qu'il n'y a jamais une situation où les deux pourraient s'employer.

Pour analyser l'effet de ces deux explications, nous les avons présentées à deux groupes d'apprenants qui par la suite ont fait deux activités, une au début du semestre avant la présentation du PC et de l'IMP et l'autre à la fin, pour mesurer leurs progrès pendant le semestre. À la fin du semestre, les étudiants ont eu le choix de se retirer de cette étude, mais aucun d'entre eux n'a choisi cette option. Les deux activités présentaient aux apprenants un conte de fées dans lequel ils devaient choisir entre PC et l'IMP pour tous les verbes. Ce genre d'activité est très commun dans la salle de classe quand les apprenants commencent à apprendre la distinction PC/IMP. La première activité vient directement du livre d'exercices qui accompagne le manuel Chez nous. Les activités sont reproduites ici :

Première activité :

La famille-ours et une petite fille curieuse I. Voici un extrait d'une histoire pour enfants que vous connaissez sans doute. Décidez si on doit mettre les verbes au passé composé ou à l'imparfait en soulignant la forme appropriée. Le premier verbe a été souligné pour vous comme exemple.

Il (1) [a été / était] une fois une famille d'ours qui (2) [a habité / habitait] une jolie maison dans les bois. Tous les matins, Maman-ours (3) [a préparé / préparait] des céréales chaudes pour sa famille. Un matin, Papa-ours (4) [a dit / disait] : «C'est trop chaud. Attendons avant de manger. » La Famille-ours (5) [a décidé / décidait] de faire une promenade dans les bois avant de manger.

De l'autre côté de la forêt, une petite fille (6) [s'est réveillée / se réveillait]. Il (7) [a fait / faisait] beau. Elle (8) [a décidé / décidait] de faire une promenade dans les bois. Elle (9) [a découvert / découvrait] la maison de la Famille-ours. Elle (10) [a ouvert / ouvrait] la porte et elle (11) [est entrée / entrait] dans la maison. Elle (12) [a eu / avait] faim (hungry). Elle (13) [a goûté / goûtait] (tasted) aux trois bols de céréales sur la table. Celui de Papaours (14) [a été / était] trop chaud. Celui de Maman-ours (15) [a été / était] trop froid. Mais celui de Bébé-ours (16) [a été / était] parfait. La petite fille (17) [a mangé / mangeait] le bol entier !

Après le petit déjeuner, elle (18) [a été / était] fatiguée, donc elle (19) [a monté / montait] l'escalier pour trouver un lit confortable. Dans la chambre, il y (20) [a eu / avait] trois lits. Elle (21) [a essayé / essayait] (tried) le lit de Bébé-ours ; (22) [ça a été / c'était] parfait. La petite fille (23) [s'est endormie / s'endormait] tout de suite. Elle (24) [a dormi / dormait] tranquillement quand les trois ours (25) [sont rentrés / rentraient] à la maison.

Deuxième activité :

Cendrillon. Voici un extrait d'une histoire pour enfants que vous connaissez sans doute. Décidez si on doit mettre les verbes au passé composé ou à l'imparfait en soulignant la forme appropriée. 
Il y (26) [a eu/avait] une fois un homme fort et riche dont la femme (27) [est tombée/tombait] malade. Se sentant près de sa fin, elle (28) [a fait/faisait] venir sa fille unique, qui (29) [a été/était] encore toute petite.

— «Ma chère enfant », elle lui (30) [a dit/disait], " sois toujours bonne et pieuse, et le bon Dieu t'aidera. »

Après quoi, ayant clos ses yeux, elle (31) [est morte/mourait].

Chaque jour la jeune fille (32) [est allée/allait] pleurer sur la tombe de sa mère, et ainsi elle (33) [est restée/restait] bonne et pieuse. Le cimetière (34) [a été/était] caché sous la neige comme sous un linceul (shroud) et, quand le soleil (35) [a fondu/fondait] (fondre = to melt) la neige, le père (36) [a pris/prenait] une autre femme.

Celle-ci (37) [a amené/amenait] dans la maison deux filles belles de figure, mais laides de cœur. Depuis ce moment, pour la pauvre enfant une vie bien dure (38) [a commencé/commençait].

— «Qu'est-ce que cette créature fait dans notre chambre ? » les belles-sœurs (39) [ont dit/disaient]. «Qui veut manger doit gagner son pain; la place de la servante est dans la cuisine. »

Elles lui (40) [ont enlevé/enlevaient] ses beaux vêtements, lui (41) [ont donné/donnaient] de vieux vêtements, et, après la (42) [ont menée/menaient] (mener = to take [someone somewhere]) dans la cuisine où elle (43) [a été/était] forcée de faire beaucoup de travail, de se lever avant l'aube, de puiser de l'eau, d'allumer le feu, d'apprêter (to prepare) les repas et la lessive.

Ses sœurs lui (44) [ont fait/faisaient] toutes les niches (tricks) possibles; elles se (45) [sont moquées/moquaient] d'elle et lui (46) [ont crié/criaient] (crier = to scream) dessus. Le soir, elle n' (47) [a pas eu/avait pas] de lit pour dormir et elle (48) [a du/devait] se coucher dans les cendres du foyer. Elle (49) [a paru/paraissait] donc toujours sale et poudreuse, et c'est pourquoi on lui (50) [a donné/donnait] le nom Cendrillon.

Chaque activité contenait 25 verbes, mais la première réponse pour la première activité était déjà donnée, donc il y avait un total de 49 verbes pour les deux activités, alors 49 réponses par participant. Dans 44 instances, les apprenants n'ont pas choisi (PC ou IMP), donc ces verbes sont exclus des résultats présentés cidessous. En fin de compte les neuf apprenants dans le groupe expérimental ont choisi 427 formes verbales et les 18 apprenants dans le groupe témoin en ont choisi 852. Si l'explication aspectuelle influence l'acquisition des apprenants de la distinction entre le PC et l'IMP, on s'attendrait à trouver que les apprenants du groupe expérimental choisissent des réponses plus similaires à celles des locutrices natives que les apprenants du groupe témoin.

Nous avons demandé à des locutrices natives de compléter les mêmes activités que les apprenants pour pouvoir par la suite comparer les réponses des apprenants à celles des locutrices natives. Les réponses des neuf locutrices natives, dont une d'Algérie, une de Côte d'Ivoire, une du Sénégal, deux du Canada, et quatre de France, n'ont pas toujours fait l'unanimité. Ces locutrices natives sont surtout des étudiantes graduées, mais qui n'étudient pas forcement la linguistique française. Comme une production aussi native que possible est le but de l'apprentissage d'une langue étrangère, on avait besoin de ce modèle natif pour déterminer si les apprenants s'approchaient de ce but.

\section{Résultats et discussion}

Pour déterminer si les étudiants ont appliqué les règles qu'on leur a présentées dans leur choix entre le PC et l'IMP, il est nécessaire de considérer chaque verbe dans les deux activités individuellement. Le Tableau 2 présente les réponses au PC pour tous les verbes dans la première activité, numérotés de 2 à 25 puisque le premier verbe servait d'exemple. 
Tableau 2: Taux de verbes au PC pour la première activité (Les pourcentages en caractères gras sont les pourcentages qui s'écartent le plus de ceux des locuteurs natifs.)

\begin{tabular}{|c|c|c|c|}
\hline Exemple & Locutrices natives & Groupe expérimental & Groupe témoin \\
\hline 2 (habiter) & $0 \%(0 / 9)$ & $67 \%(6 / 9)$ & $19 \%(3 / 16)$ \\
\hline 3 (préparer) & $0 \%(0 / 9)$ & $0 \%(0 / 9)$ & $29 \%(5 / 17)$ \\
\hline 4 (dire) & $89 \%(8 / 9)$ & $100 \%(9 / 9)$ & $78 \%(14 / 18)$ \\
\hline 5 (décider) & $89 \%(8 / 9)$ & $100 \%(9 / 9)$ & $88 \%(15 / 17)$ \\
\hline 6 (se réveiller) & $22 \%(2 / 9)$ & $67 \%(6 / 9)$ & $88 \%(15 / 17)$ \\
\hline 7 (faire) & $0 \%(0 / 9)$ & $22 \%(2 / 9)$ & $18 \%(3 / 17)$ \\
\hline 8 (décider) & $100 \%(9 / 9)$ & $100 \%(9 / 9)$ & $82 \%(14 / 17)$ \\
\hline 9 (découvrir) & $100 \%(9 / 9)$ & $100 \%(9 / 9)$ & $94 \%(16 / 17)$ \\
\hline 10 (ouvrir) & $100 \%(8 / 8)$ & $89 \%(8 / 9)$ & $82 \%(14 / 17)$ \\
\hline 11 (entrer) & $100 \%(8 / 8)$ & $75 \%(6 / 8)$ & $82 \%(14 / 17)$ \\
\hline 12 (avoir) & $0 \%(0 / 9)$ & $78 \%(7 / 9)$ & $6 \%(1 / 18)$ \\
\hline 13 (goûter) & $100 \%(9 / 9)$ & $100 \%(9 / 9)$ & $89 \%(16 / 18)$ \\
\hline 14 (être) & $0 \%(0 / 9)$ & $0 \%(0 / 9)$ & $6 \%(1 / 18)$ \\
\hline 15 (être) & $0 \%(0 / 9)$ & $0 \%(0 / 9)$ & $6 \%(1 / 17)$ \\
\hline 16 (être) & $0 \%(0 / 9)$ & $11 \%(1 / 9)$ & $11 \%(2 / 18)$ \\
\hline 17 (manger) & $100 \%(9 / 9)$ & $100 \%(9 / 9)$ & $89 \%(16 / 18)$ \\
\hline 18 (être) & $0 \%(0 / 9)$ & $0 \%(0 / 9)$ & $6 \%(1 / 17)$ \\
\hline 19 (monter) & $100 \%(9 / 9)$ & $100 \%(9 / 9)$ & $88 \%(15 / 17)$ \\
\hline 20 (avoir) & $0 \%(0 / 9)$ & $44 \%(4 / 9)$ & $25 \%(4 / 16)$ \\
\hline 21 (essayer) & $100 \%(9 / 9)$ & $89 \%(8 / 9)$ & $83 \%(15 / 18)$ \\
\hline 22 (être) & $0 \%(0 / 9)$ & $11 \%(1 / 9)$ & $6 \%(1 / 17)$ \\
\hline 23 (s'endormir) & $100 \%(9 / 9)$ & $100 \%(9 / 9)$ & $78 \%(14 / 18)$ \\
\hline 24 (dormir) & $11 \%(1 / 9)$ & $44 \%(4 / 9)$ & $28 \%(5 / 18)$ \\
\hline 25 (rentrer) & $100 \%(9 / 9)$ & $89 \%(8 / 9)$ & $82 \%(14 / 17)$ \\
\hline
\end{tabular}

Dans le Tableau 2 on observe plus de variation dans les réponses des apprenants que dans celles des locutrices natives. Les locutrices ont toutes choisi la même réponse sauf pour quatre verbes sur 24 (les numéros 4, 5, 6, et 24) pour un total de 5 réponses seulement qui s'écartent de la réponse majoritaire. Pour le groupe expérimental il n'y a pas l'unanimité de réponse pour 12 verbes sur 24 avec 25 réponses minoritaires en tout et pour le groupe témoin ces chiffres sont 24 verbes sur 24 et 61 réponses minoritaires. La plus grande différence entre les réponses du groupe témoin est moins surprenante quand on se rappelle qu'il y a 18 personnes dans le groupe témoin contre neuf dans le groupe expérimental et neuf locutrices natives. 
Le Tableau 3 montre les résultats pour la deuxième activité. Encore une fois, les pourcentages sont des pourcentages pour l'emploi du PC.

Tableau 3: Taux de verbes au PC pour la deuxième activité (Les pourcentages en caractères gras sont les pourcentages qui s'écartent le plus de ceux des locuteurs natifs.)

\begin{tabular}{|c|c|c|c|}
\hline Exemple & Locutrices natives & Groupe expérimental & Groupe témoin \\
\hline 26 (avoir) & $11 \%(1 / 9)$ & $13 \%(1 / 8)$ & $29 \%(5 / 17)$ \\
\hline 27 (tomber) & $100 \%(9 / 9)$ & $75 \%(7 / 9)$ & $72 \%(13 / 18)$ \\
\hline 28 (faire) & $100 \%(9 / 9)$ & $43 \%(3 / 7)$ & $75 \%(12 / 16)$ \\
\hline 29 (être) & $0 \%(0 / 9)$ & $22 \%(2 / 9)$ & $6 \%(1 / 16)$ \\
\hline 30 (dire) & $100 \%(9 / 9)$ & $89 \%(8 / 9)$ & $100 \%(17 / 17)$ \\
\hline 31 (mourir) & $100 \%(8 / 8)$ & $100 \%(9 / 9)$ & $89 \%(16 / 18)$ \\
\hline 32 (aller) & $11 \%(1 / 9)$ & $0 \%(0 / 9)$ & $11 \%(2 / 18)$ \\
\hline 33 (rester) & $67 \%(6 / 9)$ & $22 \%(2 / 9)$ & $18 \%(3 / 17)$ \\
\hline 34 (être) & $0 \%(0 / 9)$ & $0 \%(0 / 8)$ & $19 \%(3 / 16)$ \\
\hline 35 (fondre) & $89 \%(8 / 9)$ & $78 \%(7 / 9)$ & $82 \%(14 / 17)$ \\
\hline 36 (prendre) & $100 \%(9 / 9)$ & $89 \%(8 / 9)$ & $82 \%(14 / 17)$ \\
\hline 37 (amener) & $100 \%(9 / 9)$ & $56 \%(5 / 9)$ & $67 \%(12 / 18)$ \\
\hline 38 (commencer) & $78 \%(7 / 9)$ & $89 \%(8 / 9)$ & $61 \%(11 / 18)$ \\
\hline 39 (dire) & $56 \%(5 / 9)$ & $100 \%(9 / 9)$ & $94 \%(17 / 18)$ \\
\hline 40 (enlever) & $78 \%(7 / 9)$ & $88 \%(7 / 8)$ & $56 \%(10 / 18)$ \\
\hline 41 (donner) & $75 \%(6 / 8)$ & $88 \%(7 / 8)$ & $65 \%(11 / 17)$ \\
\hline 42 (mener) & $78 \%(7 / 9)$ & $86 \%(6 / 7)$ & $72 \%(13 / 18)$ \\
\hline 43 (être) & $25 \%(2 / 8)$ & $25 \%(2 / 8)$ & $39 \%(7 / 18)$ \\
\hline 44 (faire) & $25 \%(2 / 8)$ & $50 \%(4 / 8)$ & $39 \%(7 / 18)$ \\
\hline 45 (se moquer) & $11 \%(1 / 9)$ & $63 \%(5 / 8)$ & $33 \%(6 / 18)$ \\
\hline 46 (crier) & $11 \%(1 / 9)$ & $56 \%(5 / 9)$ & $47 \%(8 / 17)$ \\
\hline 47 (avoir) & $0 \%(0 / 9)$ & $44 \%(4 / 9)$ & $33 \%(3 / 18)$ \\
\hline 48 (devoir) & $11 \%(1 / 9)$ & $38 \%(3 / 8)$ & $22 \%(4 / 18)$ \\
\hline 49 (paraître) & $0 \%(0 / 9)$ & $13 \%(1 / 8)$ & $11 \%(2 / 18)$ \\
\hline 50 (donner) & $67 \%(6 / 9)$ & $78 \%(7 / 9)$ & $72 \%(13 / 18)$ \\
\hline
\end{tabular}

Ce qui saute aux yeux dans le Tableau 3 est la plus grande diversité de réponses chez les locutrices natives dans la deuxième activité que dans la première. Il n'y a que dix verbes sur 25 pour lesquels le choix du PC ou l'IMP est catégorique (les numéros 27, 28, 29, 30, 31, 34, 36, 37, 47, et 49) et 27 réponses en tout qui 
s'écartent de la norme. Pour le groupe expérimental il n'y a pas unanimité pour 21 verbes sur 25 avec 46 réponses minoritaires en tout et pour le groupe témoin ces chiffres montent à 24 verbes sur 25 et 97 réponses minoritaires.

Quand on regarde individuellement les verbes qui ont changé de réponse pour chaque groupe, on voit l'influence des deux explications grammaticales. Comme les deux activités ne contiennent pas les mêmes verbes, on va considérer seulement les verbes en commun dans les deux activités pour lesquels les apprenants et les locutrices natives ne choisissent pas le même temps verbal. Par exemple, le verbe être est presque toujours à l'IMP chez les locutrices natives: à $100 \%$ dans la première activité (verbes $14,15,16$, et 22) et à $92 \%(24 / 26)$ dans la deuxième activité (verbes 29, 34, et 43). L'exemple (43), Elle [a étélétait] forcée de faire beaucoup de travail, est le seul où deux locutrices natives ont choisi d'employer le PC. Le Tableau 4 montre les réponses pour ce verbe en comparaison avec celles des deux groupes d'apprenants.

Tableau 4 : Taux d'être à l'imparfait pour chaque groupe et chaque activité

\begin{tabular}{|l|l|l|l|}
\hline & Locutrices natives & Groupe expérimental & Groupe témoin \\
\hline première activité & $100 \%(45 / 45)$ & $96 \%(43 / 45)$ & $93 \%(81 / 87)$ \\
\hline deuxième activité & $92 \%(24 / 26)$ & $84 \%(21 / 25)$ & $78 \%(39 / 50)$ \\
\hline
\end{tabular}

Dans le Tableau 4 on voit que, comme les locutrices natives, les étudiants ont choisi le PC pour le verbe être plus souvent dans la deuxième activité. Les réponses du groupe expérimental sont les plus similaires à celles des locutrices natives. Ils choisissent l'IMP pour le verbe être avec seulement deux exceptions (verbes 16 et 22) dans la première activité et dans la deuxième quatre étudiants ont choisi le PC pour le verbe 43 tout comme deux des locutrices natives. En ce qui concerne le groupe témoin, on voit que ces apprenants ont choisi surtout l'IMP comme les locutrices natives dans la première activité avec six exceptions, mais ils ont choisi l'IMP dans seulement $78 \%$ des cas pour la deuxième. Il y a 11 exemples en tout où ces apprenants n'ont pas choisi l'IMP dans la deuxième activité. Sept parmi ces 11 sont pour le verbe 43 pour lequel deux locutrices natives ont choisi le PC aussi, mais un est dans l'exemple (29), sa fille [...] [a été/était] encore toute petite, et trois sont dans l'exemple (34), Le cimetière [a étélétait] caché sous la neige. Il n'y a rien dans l'explication grammaticale du PC de Chez nous qui puisse motiver le choix du PC chez ces apprenants sauf s'ils voient (29) comme quelque chose qui avance la narration et (34) comme un événement achevé. Les réponses des apprenants du groupe expérimental, alors, s'approchent plus de celles des locutrices natives pour le verbe être.

Le deuxième verbe qu'on va considérer est le verbe avoir. Il y a deux occurrences d'avoir dans chaque activité : les verbes (12) et (20) dans la première et (26) et (47) dans la deuxième. Dans la première activité, toutes les locutrices ont toujours choisi l'IMP pour ce verbe (18/18) et pour la deuxième seulement une locutrice native a choisi le PC pour une occurrence d'avoir pour un taux d'IMP de 94\% (17/18). Par contre, les apprenants ont choisi bien plus souvent le PC pour le verbe avoir. Le Tableau 5 montre la comparaison entre les groupes d'apprenants et les locutrices natives pour le verbe avoir.

Tableau 5 : Taux d'avoir à l'imparfait pour chaque groupe et chaque activité

\begin{tabular}{|l|l|l|l|}
\hline & Locutrices natives & Groupe expérimental & Groupe témoin \\
\hline première activité & $100 \%(18 / 18)$ & $67 \%(12 / 18)$ & $85 \%(29 / 34)$ \\
\hline deuxième activité & $94 \%(17 / 18)$ & $71 \%(12 / 17)$ & $77 \%(27 / 35)$ \\
\hline
\end{tabular}

En ce qui concerne le groupe expérimental, on voit qu'il y a beaucoup de variation dans leurs choix entre le PC et l'IMP bien que les choix des locutrices natives ne varient presque pas. Deux étudiants du groupe 
expérimental ont choisi le PC pour le verbe (12), Elle [a eu/avait] faim, de la première activité. Ce verbe se trouve après beaucoup de verbes qui sont au PC selon les locutrices natives, alors il est possible que les apprenants de ce groupe aient choisi le PC parce que les verbes qui le précèdent sont au PC. Le groupe témoin, par contre, a choisi l'IMP dans ce contexte comme les locutrices natives à une exception près. Pour le verbe (20), il y [a eu / avait] trois lits, quatre étudiants de chacun des deux groupes a choisi le PC. Dans la deuxième activité, les deux groupes d'apprenants ont choisi le PC plus souvent que les locutrices natives. Ceci est le cas de l'exemple (47), le soir elle n' [a pas eu/avait pas] de lit pour dormir, pour lequel le groupe expérimental a choisi le PC 4 fois sur 9 et le groupe témoin l'a fait 3 fois sur 18 tandis que les locutrices natives ont toujours choisi l'IMP. On peut supposer que quelques apprenants du groupe expérimental voyaient ce verbe comme le début d'un état, un emploi qui favorise le PC selon l'explication aspectuelle. Pour les apprenants du groupe témoin, on peut supposer que ces apprenants voyaient cet événement comme une action dans une narration. Tout compris, aucun des groupes n'est d'accord avec les locutrices natives.

Jusqu'ici, on a considéré deux verbes d'état, être et avoir. Quelles ont été donc les choix entre PC et IMP pour les verbes d'action? Pour répondre à cette question, on va comparer les verbes (6) se réveiller et (24) dormir de la première activité et (33) rester et (39) dire de la deuxième puisque ces verbes sont ceux pour lesquels les locutrices natives et les apprenants ont choisi des temps de verbe différents. Le Tableau 6 montre les différences entre les trois groupes en ce qui concerne ces verbes. Cette fois, les pourcentages sont du PC et non pas de l'IMP.

Tableau 6: Taux des verbes (6) se réveiller, (24) dormir, (33) rester et (39) dire au PC

\begin{tabular}{|l|l|l|l|}
\hline & Locutrices natives & Groupe expérimental & Groupe témoin \\
\hline$(6)$ se réveiller & $22 \%(2 / 9)$ & $67 \%(6 / 9)$ & $88 \%(15 / 17)$ \\
\hline$(24)$ dormir & $11 \%(1 / 9)$ & $44 \%(4 / 9)$ & $28 \%(5 / 18)$ \\
\hline$(33)$ rester & $67 \%(6 / 9)$ & $22 \%(2 / 9)$ & $18 \%(3 / 17)$ \\
\hline$(39)$ dire & $56 \%(5 / 9)$ & $100 \%(9 / 9)$ & $94 \%(17 / 18)$ \\
\hline
\end{tabular}

Dans le Tableau 6, on voit que tous les groupes varient leurs réponses pour ces quatre verbes. Pour les verbes de la première activité les contextes sont les suivants : (6) De l'autre côté de la forêt, une petite fille [s'est réveillée/se réveillait] et (24) Elle [a dormi/dormait] tranquillement quand les trois ours [sont rentrés/rentraient] à la maison. Pour ces deux verbes, les locutrices natives choisissent l'IMP plus souvent que le PC, et les apprenants font le contraire. Il faut noter que les deux formes verbales sont possibles ici, selon les locutrices natives.

Pour les verbes de la deuxième activité, on voit un effet clair de l'explication grammaticale. De nouveau, il faut signaler que, selon les locutrices natives, et le PC et l'IMP sont possibles. Voici les contextes de ces deux verbes: (33) Chaque jour la jeune fille allait pleurer sur la tombe se sa mère, et ainsi elle [est restée/restait] bonne et pieuse et les belles-sœurs [ont dit/disaient] "Qui veut manger dit gagner son pain [...] ». Pour le verbe (33) rester la plupart des locutrices natives ont choisi l'IMP tandis que la plupart des apprenants des deux groupes ont choisi le PC. On peut supposer que les apprenants du groupe expérimental voyaient la situation comme une réponse à la question Qu'est-ce qui s'est passé ? tandis que le groupe témoin la voyait comme une action achevée.

Le verbe dire est où on voit le plus clairement l'influence de l'instruction. Cinq locutrices natives sur 9 ont choisi le PC tandis que presque tous les apprenants des deux groupes ont choisi le PC. De nouveau, bien que les deux formes verbales soient possibles, les apprenants voyaient ce verbe comme un événement qui s'est passé ou une action achevée. 


\section{Conclusion}

Cette étude a comparé deux méthodes d'enseignement du système aspectuel du français, une qui est aspectuelle (basée sur Dansereau, 1987 et Frantzen, 1995) et une qui vient du manuel Chez nous, par le biais de deux activités dans lesquelles deux groupes d'apprenants ont choisi d'employer le PC ou l'IMP pour 49 verbes. L'analyse de six de ces verbes, avoir, être, se réveiller, dormir, rester et dire, a montré des différences entre les deux groupes d'apprenants et entre les apprenants et les locutrices natives qui ont complété ces mêmes activités, mais il n'est pas encore clair quel a été l'effet des deux explications proposées aux apprenants sur leurs choix entre le PC et l'IMP. Nous pensons que l'explication aspectuelle a ses mérites, puisque c'est celle qui représente le mieux la différence entre le PC et l'IMP. Comme Dansereau (1987:38) a écrit, « having presented the passé compose/imparfait distinction [as aspectual], the instructor must stick to this definition at all times, stressing the importance of the students' perception of the event. He must never fall back on any of the traditional terminology, such as "duration" or "repetition," which has been shown to be at best confusing and at worst inaccurate. [...] And then, for the rest, the students must do many, many exercises. » Il faut se rappeler qu'apprendre une langue exige beaucoup de dévouement, beaucoup de temps et beaucoup de pratique ce qui explique peut-être pourquoi on n'a pas trouvé de résultats qui soutiennent clairement la supériorité de l'explication aspectuelle en un seul semestre. Pour examiner plus profondément l'influence des explications, il faut l'examiner au travers de plusieurs semestres et années.

Il faut considérer aussi le type d'activité que ces apprenants ont fait pour cette étude. S'il est vrai que la distinction aspectuelle dépend du point de vue du locuteur, ces activités, mêmes si elles sont très communes dans la salle de classe de FLE, ne sont pas la meilleure façon de tester la compréhension de la distinction aspectuelle chez les apprenants. Comme Blyth (1997 : 62) le signale, « a cloze passage [un exercice à trous] and third person narratives are mediated by someone else's subjectivity and thus are inherently problematic. [...] A cloze passage essentially requires the student to reconstruct the mental processes of someone else's mind. ». Cette idée est en fait cruciale car la définition dite "aspectuelle » est fondée sûr l'intention du scripteur. Si on ne connaît pas l'intention du scripteur dans un texte déjà écrit, il est impossible que les apprenants sachent choisir entre deux choix aspectuelles. Ceci est vrai surtout quand les deux formes sont possibles, même si elles ne correspondent pas à un même sens. Nous avons l'intention dans une étude en préparation de comparer l'emploi du PC et de l'IMP par les apprenants des deux groupes dans des compositions libres. Notre hypothèse est que la différence entre les emplois du PC et de l'IMP sera plus grande entre les deux groupes pour cette activité que pour les activités considérées dans la présente étude.

\section{Références bibliographiques}

Andersen, R. W. (1991). Development sequences: The Emergence of aspect marking in second language acquisition. In Huebner, T. et Ferguson, C. A. (éd.), Crosscurrents in second language acquisition and linguistic theories, Amsterdam/Philadelphia: John Benjamins, 305-24.

Bardovi-Harlig, K. (2000). Tense and aspect in second language acquisition: Form, meaning, and use. Malden, MA/Oxford, UK: Blackwell.

Blyth, C. (1997). A Constructivist approach to grammar: Teaching teachers to teach aspect. The Modern Language Journal, 81, 50-66.

Chastain, K. (1987). Examining the role of grammar explanations, drills, and exercises in the development of communication skills. Hispania, 70, 160-66.

Comrie, B. (1976). Aspect. London/New York: Cambridge University Press.

Dansereau, D. (1987). A Discussion of techniques used in the teaching of the passé composé/imparfait distinction in French. French Review, 61, 33-38. 
Frantzen, D. (1995). Preterite/Imperfect half-truths: Problems with Spanish textbook rules for usage. Hispania, 78, 14558.

Guitart, J. M. (1978). Aspects of Spanish aspect: A New look at the preterit/imperfect distinction. In Suñer, M. (éd.), Contemporary studies in Romance Linguistics, Georgetown: Georgetown University Press, 132-68.

Labelle, M. (1987). L’Utilisation des temps du passé dans les narrations françaises: Le passé composé, l'imparfait, et le présent historique. Revue Romane, 22, 3-29.

López-Ortega, N. (2000). Tense, aspect, and narrative structure in Spanish as a second language. Hispania, 83, 488-502.

Lunn, P. (1985). The Aspectual Lens. Hispanic Linguistics, 2, 49-61.

Pons, C., Sculle, M. E., et Valdman, A. (2006). Chez nous. Upper Saddle River, NJ: Pearson Education.

Smith, C. S. (1983). A Theory of aspectual choice. Language, 59, 479-501.

Vendler, Z. (1967). Linguistics in Philosophy. Ithaca, NY: Cornell University Press.

Weinrich, H. (1964). Tempus: Besprochene und erzählte Welt. Stuttgart, Allemagne: W. Kohlhammer GmbH.

Westfall, R. et Foerster, S. (1996). Beyond aspect: New strategies for teaching the preterit and the imperfect. Hispania, 79, 550-60. 